\title{
A coverslip-based technique for evaluating Staphylococcus aureus biofilm formation on human plasma
}

\author{
Jennifer N. Walker and Alexander R. Horswill *
}

Department of Microbiology, Roy J. and Lucille A. Carver College of Medicine, University of lowa, lowa City, IA, USA

\section{Edited by:}

David Heinrichs, University of Western Ontario, Canada

Reviewed by:

Odile Tresse, French National Institute for Agricultural

Research/Nantes-Atlantic National

College of Veterinary Medicine,

Food Science and Engineering

France

Julie Morrissey, University of

Leicester, UK

\section{${ }^{*}$ Correspondence:}

Alexander R. Horswill, Department of Microbiology, University of lowa, 540F EMRB, lowa City, IA 52242,

USA.

e-mail: alex-horswill@uiowa.edu
The ability of the opportunistic pathogen, Staphylococcus aureus, to form biofilms is increasingly being viewed as an important contributor to chronic infections. In vitro methods for analyzing $S$. aureus biofilm formation have focused on bacterial attachment and accumulation on abiotic surfaces, such as in microtiter plate and flow cell assays. Microtiter plates provide a rapid measure of relative biomass levels, while flow cells have limited experimental throughput but are superior for confocal microscopy biofilm visualization. Although these assays have proven effective at identifying mechanisms involved in cell attachment and biofilm accumulation, the significance of these assays in vivo remains unclear. Studies have shown that when medical devices are implanted they are coated with host factors, such as matrix proteins, that facilitate $S$. aureus attachment and biofilm formation. To address the challenge of integrating existing biofilm assay features with a biotic surface, we have established an in vitro biofilm technique utilizing UV-sterilized coverslips coated with human plasma. The substratum more closely resembles the in vivo state and provides a platform for $S$. aureus to establish a robust biofilm. Importantly, these coverslips are amenable to confocal microscopy imaging to provide a visual reference of the biofilm growth stage, effectively merging the benefits of the microtiter and flow cell assays. We confirmed the approach using clinical $S$. aureus isolates and mutants with known biofilm phenotypes. Altogether, this new biofilm assay can be used to assess the function of $S$. aureus virulence factors associated with biofilm formation and for monitoring the efficacy of biofilm treatment modalities.

Keywords: Staphylococcus aureus, MRSA, biofilm, assay

\section{INTRODUCTION}

Staphylococcus aureus is a Gram-positive bacterial pathogen and a major public health problem in the United States and other countries. S. aureus causes a wide range of infections that includes acute, chronic, and toxin mediated disease (Chambers and DeLeo, 2009; DeLeo and Chambers, 2009). The ability of this pathogen to cause such a diverse array of problems is due to its arsenal of virulence factors that include pore-forming toxins, superantigens, matrix-binding surface adhesins, and tissue-degrading enzymes (Lowy, 1998). Like many bacterial pathogens, S. aureus can attach to a surface, accumulate biomass, and form a community of cells encased in an extracellular matrix called a biofilm (Gotz, 2002; Kiedrowski and Horswill, 2011). Many chronic infections, such as endocarditis, osteomyelitis, and growth on medical implants have biofilm-like features (Parsek and Singh, 2003; Zimmerli et al., 2004; Costerton, 2005). The formation of biofilms has been shown to protect the bacteria from host immune defenses as well as antibiotic therapy (Zimmerli et al., 2004; Costerton, 2005; Hall-Stoodley and Stoodley, 2005; del Pozo and Patel, 2007), and they remain an ongoing challenge for treating chronic infections. Although numerous factors have been demonstrated to be essential for $S$. aureus biofilm development, much remains unknown about the specific mechanisms employed to form and dissemble biofilm communities (Boles and Horswill, 2011; Kiedrowski and Horswill, 2011).
Many in vitro assays have been developed to quantify S. aureus biofilm formation (Cassat et al., 2007). Perhaps the most commonly used assay is the direct attachment of the bacteria to uncoated, polystyrene microtiter plates. While this assay is convenient and low-cost, it is becoming evident that many clinical S. aureus isolates attach poorly to abiotic surfaces (Lauderdale et al., 2009; Beenken et al., 2010). Studies examining medical implants indicate that they are coated with host matrix proteins upon implantation (Francois et al., 1998, 2000), and these host matrices can serve as a handle that are bound by the numerous proteins coating the S. aureus surface (Foster and Hook, 1998), facilitating cell attachment and biofilm accumulation. Matrix proteins can be coated on microtiter plates to more accurately reproduce in vivo-like conditions (Cassat et al., 2007), and in side-by-side comparisons, this strategy accentuates $S$. aureus biofilm formation (Beenken et al., 2010). These matrix-coated plates are convenient for biofilm assays, but they are not ideal in the experimental transition to confocal microscopy for obtaining a visual representation of the biofilm structure. Flow cells continue to be the method of choice for in situ microscopy, but they are laborious to perform, amenable to only limited experimental throughput, and require large quantities of media that can exhaust valuable reagents.

Although the microtiter and flow cells assays are the most commonly used methods to assess $S$. aureus biofilm formation 
(Cassat et al., 2007), other related assays have been developed to examine pathogens such as Enterococcus faecalis and Pseudomonas aeruginosa during biofilm formation (Goeres et al., 2009; Guiton et al., 2009). These assays utilize a variety of surfaces for bacterial attachment to assess biofilm formation through microscopy, staining of the biomass, or viable plate counts. Our goal in this work was to develop a hybrid biofilm method that drew upon the benefits of both microtiter-based assays and flow cells. Based on success in Enterococcus faecalis biofilm studies with coverslips as a substratum (Guiton et al., 2009), we developed a coverslip-based approach amenable to $S$. aureus biofilm studies. Using clinical isolates and strains with characterized mutations, we demonstrated that the new method is robust and mirrors results obtained with other biofilm assays.

\section{MATERIALS AND METHODS STRAINS AND GROWTH CONDITIONS}

The bacterial strain used in this study was a communityassociated methicillin-resistant $S$. aureus (CA-MRSA) isolate of the USA300 pulse field gel group called LAC, hereafter called "LAC WT." As a negative control, we used a sigma factor B (SigB) deficient mutant, hereafter called "LAC $\Delta$ sigB" (Lauderdale et al., 2009). Additional control strains used in this study include the USA400 clinical isolate MW2 (Baba et al., 2002) and a LAC strain with a complete deletion of the ica locus (Lauderdale et al., 2009). The biofilm phenotypes of LAC WT, LAC $\Delta i c a$, LAC $\Delta s i g B$, and the construction of the $\Delta \operatorname{sig} B$ and the $\Delta i c a$ mutations, were reported previously (Lauderdale et al., 2009). S. aureus strains were maintained in tryptic soy broth (TSB) or brain heart infusion broth (BHI) throughout this work.

\section{PREPARATION OF BIOFILM MICROTITER PLATES}

$22 \mathrm{~mm} \times 22 \mathrm{~mm}$ polyvinyl plastic coverslips (Raylabcon, cat \#20 $405300)$ were placed in each well of a six well cell culture plate (Corning Costar, cat \# 3516). Plates with coverslips were then UV-sterilized in a tissue culture hood for at least $2 \mathrm{~h}$. Heparin was added 1:1 to human plasma prepared from donors at the University of Iowa Inflammation Program to prevent clotting. The prepared human plasma was then diluted in $50 \mathrm{mM}$ sodium bicarbonate solution to a final concentration of $20 \%$. A $2 \mathrm{ml}$ aliquot of either the sodium bicarbonate buffer or the $20 \%$ human plasma solution was added to each of the sterilized wells with coverslips and incubated at $4^{\circ} \mathrm{C}$ overnight. The human plasma was aspirated off the next day, and the wells and coverslips were washed twice with $2 \mathrm{ml}$ sterile water.

\section{BIOFILM CONDITIONS}

Biofilms were grown by subculturing $18 \mathrm{~h}$ cultures to a starting $\mathrm{OD}_{600}$ of 0.2 and immediately diluting them 1:100 in BHI supplemented with $0.4 \%$ glucose. A $5 \mathrm{ml}$ aliquot of each strain was added to each of the 3 wells previously coated with human plasma. Biofilms were allowed to shake at $100 \mathrm{rpm}$ at $37^{\circ} \mathrm{C}$ for $18 \mathrm{~h}$. To harvest the biofilms, the media was aspirated off and the biomass was washed twice with $5 \mathrm{ml}$ of sterile water. The biomass was allowed to air dry and stained for quantification. To assess biomass, biofilms were stained with $500 \mu \mathrm{l}$ of $0.1 \%$ crystal violet for $10 \mathrm{~min}$ at room temperature. Excess dye was removed and biofilms were washed with $5 \mathrm{ml}$ of sterile water. Before solubilization, coverslips were imaged on a Chemi Gel Doc 2000 imaging station (Bio-Rad Life Sciences, Hercules, CA). Biofilms were airdried and the biomass was dissolved in $500 \mu 133 \%$ acetic acid. A $100 \mu \mathrm{l}$ aliquot was transferred to a 96 well plate and measured on an Infinite M200 Tecan plate reader at an optical density (OD) of 635. Biomass was calculated by averaging three coverslips per strain, and at least seven separate experiments were performed with similar results.

\section{CONFOCAL MICROSCOPY}

All images were captured on a Nikon ECLIPSE C1 confocal laser scanning microscope (Nikon Inc., Melville, NY) using a $40 \times$ lens. Images were captured using the laser scanning $\mathrm{C} 1$ software from Nikon Inc. For microscopy, biofilms were harvested as described above and stained with SYTO9 (Invitrogen) and TO-PRO3 (Invitrogen) for $10 \mathrm{~min}$ in the dark. To prepare the stain, $1 \mu \mathrm{l}$ of SYTO9 was mixed with $1 \mu$ l of TO-PRO3 from the manufacturer's stocks in $1 \mathrm{ml}$ of water. Excess stain was removed by washing with $5 \mathrm{ml}$ of sterile water. Coverslips were mounted on glass slides using mounting media made from $2.4 \mathrm{~g}$ polyvinyl alcohol, $12 \mathrm{ml}$ $0.2 \mathrm{M}$ Tris base, $6 \mathrm{~g}$ glycerol, and 2.5\% DABCO (Sigma-Aldrich, St. Louis, MO) in $25 \mathrm{ml} \mathrm{H} \mathrm{H}_{2} \mathrm{O}$ and kept at $4^{\circ} \mathrm{C}$ until microscopy was performed. Microscopy was performed with three coverslips per strain with at least four separate experiments.

\section{RESULTS AND DISCUSSION DEVELOPMENT OF THE COVERSLIP BIOFILM ASSAY}

Using studies on E. faecalis biofilms as a guide (Guiton et al., 2009), we developed a coverslip biofilm method for S. aureus. To facilitate cell adherence and biofilm growth of clinical strains and to develop an assay that more closely resembles an in vivo state, the coverslips were coated with human plasma to provide matrix proteins for $S$. aureus attachment. As a testing strain, we used LAC WT, which is known to form biofilms in flow cells (Lauderdale et al., 2009, 2010), but attaches poorly to unconditioned microtiter plates. After $18 \mathrm{~h}$ of growth, LAC WT formed thick and confluent biofilm structures on the coverslip coated with $20 \%$ human plasma. The coverslips were stained with $0.1 \%$ crystal violet, and images revealed dark, confluent staining across the surface indicating robust biofilm formation (Figure 1A). To determine the ability of these strains to attach specifically to plastic, LAC WT was grown on coverslips without plasma coating and the staining was markedly reduced (Figure 1B). Biofilm formation on these coverslips can be quantified through solubilization of crystal violet and measurement of OD. For the LAC WT biofilm, quantification with plasma coating revealed a significant increase vs. uncoated coverslips $\left(\mathrm{OD}_{635}\right.$ of 1.3 vs 0.35 ; Figure 1C).

As a biofilm negative control, we tested the LAC $\triangle$ sigB mutant strain, which is deficient in biofilm formation in microtiter assays and flow cells (Lauderdale et al., 2009; Boles et al., 2010). The LAC $\triangle \operatorname{sig} B$ mutant revealed minimal staining across the coverslips coated with $20 \%$ human plasma, suggestive of poor biofilm forming capacity (Figure 1A), and the low OD (0.15 at $\left.\mathrm{OD}_{635}\right)$ supported this assessment (Figure 1C). In previous studies with flow cells (Lauderdale et al., 2009), and plasma-coated microtiter 


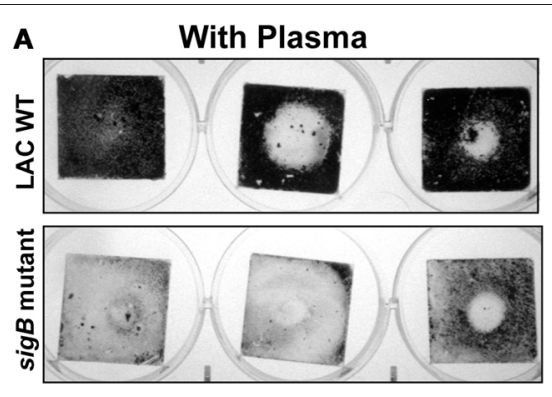

C

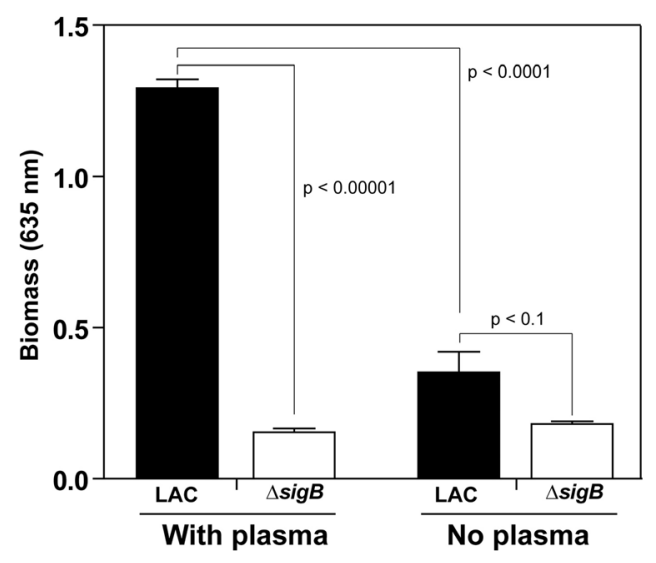

FIGURE 1 | Visualization and quantitation of coverslip biofilms. Biofilms were formed on plastic coverslips coated with $20 \%$ human plasma (A) or left untreated (B) for LAC WT and LAC $\triangle$ sigB mutant. The coverslips were post-stained with crystal violet and images were taken. (C) Average

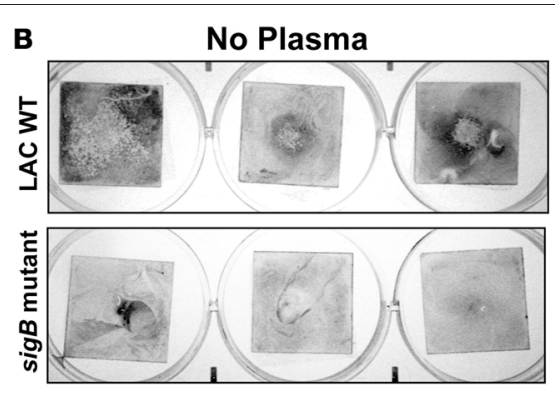

D

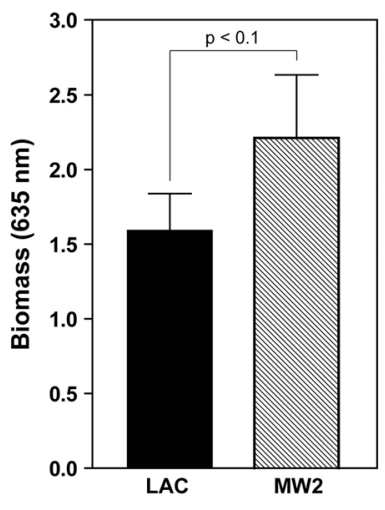

measured biomass for plasma-coated or untreated coverslip biofilms for LAC WT and $\triangle \operatorname{sig} B$ (C) or USA400 strain MW2 compared with LAC WT (D). In both (C) and (D), biomass was measured at $\mathrm{OD}_{635}$ and $P$ values were determined by students two-tailed $T$-test. plates (Mootz and Horswill, 2012), the LAC $\Delta$ sigB mutant is defective in biofilm formation, which is consistent with our coverslip assay observations. As anticipated, the LAC $\Delta$ sigB mutant also developed a poor biofilm on uncoated plastic (Figure 1B) with a measured $\mathrm{OD}_{635}$ of 0.18 (Figure 1C). The plasma-only control wells had no bacterial growth or measurable biomass (data not shown).

To confirm the generality of the new biofilm method, the clinical isolate MW2 (USA400 PFGE) was grown on plasmacoated coverslips alongside LAC WT. Post staining revealed that the MW2 strain formed thick biofilms with an average biomass of 2.2 at $\mathrm{OD}_{635}$ (Figure 1D), which was slightly higher than the LAC WT control. This observation is consistent with previous experiments where MW2 accumulates more biomass than USA300 strains (Kiedrowski et al., 2011). As another test of the new method, an LAC strain derivative with the $\Delta i c a:$ Tet deletion was examined, and this mutation eliminates the ability of S. aureus to synthesize the polysaccharide intercellular adhesin (Cramton et al., 1999). In previous flow cell biofilm experiments, we observed that introduction of the $\Delta i c a$ deletion into strain LAC did not generate an in vitro biofilm phenotype (Lauderdale et al., 2009). Similar to the previous findings, the LAC $\Delta i c a$ formed robust biofilms similar to LAC WT (data not shown), again confirming that the new coverslip assay provides an effective means to assess $S$. aureus biofilm formation.

\section{CLSM OF COVERSLIP BIOFILMS}

An important advantage to the coverslip method is the feasibility of confocal microscopy imaging of the biofilms. To prepare samples for imaging, the biofilms were grown and harvested as described above, and the coverslips were subjected to Live/Dead staining using SYTO9 and TO-PRO3 dyes and a $z$ series of images was collected by confocal laser scanning microscopy (CLSM). The image analysis of LAC WT grown on $20 \%$ human plasma revealed thick, structured biofilms with profuse staining of live cells and relatively few dead cells (Figure 2A). CLSM of LAC WT grown on uncoated coverslips formed considerably thinner biofilms with irregular structures (Figure 2B). While the LAC strain can form robust biofilm structures in flow cells on abiotic surfaces (Lauderdale et al., 2009, 2010), the limited amount of biomass accumulating on the uncoated coverslips observed here mimics previous reports with microtiter plates (Beenken et al., 2010), indicating optimal biofilm formation in this assay is strongly dependent on surface conditioning. Previous reports comparing $S$. aureus biofilm structure on uncoated vs. heparincoated coverslips also noted the strict dependence on surface modification to induce biofilm formation (Shanks et al., 2005). CLSM confirmed LAC $\triangle$ sigB grown either on plastic or on $20 \%$ human plasma-coated coverslips established poor biofilms with little structural architecture (Figures 2A,B), supporting previous flow cell observations with sigB mutants (Lauderdale et al., 2009). 


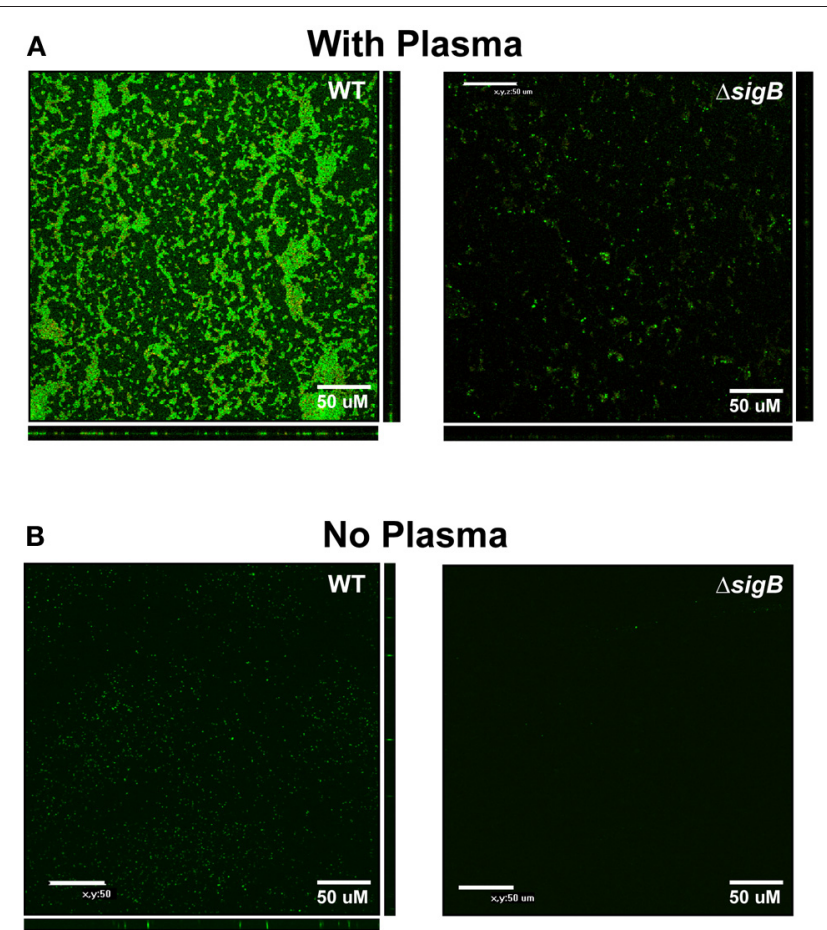

FIGURE 2 | CLSM Images of coverslip biofilms. LAC WT and LAC $\triangle$ sigB mutant biofilms were grown on human plasma-coated coverslips (A) or untreated coverslips (B). The biofilm were post-stained with SYTO9 (green) and TO-PRO3 (red), and a $z$ series of images was obtained with CLSM to visualize biofilm capacity. Each image shows a larger top down $(X Y)$ view with cross-section views of biofilm depth on the right $(Y Z)$ and bottom $(X Z)$. Only top down $(X Y)$ images were taken of LAC $\triangle$ sigB grown on plastic due to the lack of cell adherence. Biofilms did not form in the media only control wells (not shown).

Further analysis of the imaging revealed that only small clumps of live cells were attached to the slide surface.

\section{CONCLUSIONS}

The ability of $S$. aureus to attach to surfaces and develop biofilm communities complicates chronic infections, making them difficult to treat. Understanding the mechanisms employed to form

\section{REFERENCES}

Baba, T., Takeuchi, F., Kuroda, M., Yuzawa, H., Aoki, K., Oguchi, A., Nagai, Y., Iwama, N., Asano, K., Naimi, T., Kuroda, H., Cui, L., Yamamoto, K., and Hiramatsu, K. (2002). Genome and virulence determinants of high virulence community-acquired MRSA. Lancet 359, 1819-1827.

Beenken, K. E., Mrak, L. N., Griffin, L. M., Zielinska, A. K., Shaw, L. N., Rice, K. C., Horswill, A. R., Bayles, K. W., and Smeltzer, M. S. (2010). Epistatic relationships between sarA and agr in Staphylococcus aureus biofilm formation. PLoS One 5:e10790. doi: 10.1371/journal.pone.0010790
Boles, B. R., and Horswill, A. R. (2011). Staphylococcal biofilm disassembly. Trends in Microbiol. 19, 449-455.

Boles, B. R., Thoendel, M., Roth, A. J., and Horswill, A. R. (2010). Identification of genes involved in polysaccharide-independent Staphylococcus aureus biofilm formation. PLoS One 5:e10146. doi: 10.1371/journal.pone.0010146

Cassat, J. E., Lee, C. Y., and Smeltzer, M. S. (2007). Investigation of biofilm formation in clinical isolates of Staphylococcus aureus. Methods Mol. Biol. 391, 127-144.

Chambers, H. F., and DeLeo, F. R. (2009). Waves of resistance: Staphylococcus aureus in the

biofilms is essential for developing better treatment regimens, and one valuable laboratory tool to define these mechanisms has been the microtiter assay. High-throughput microtiter assays are a convenient and cost effective strategy to screen clinical isolates or mutants for biofilm capacity, but the relevance of the observations and translation in vivo is sometimes questioned. Flow cells could be a more biologically relevant mimic of the in vivo situation, but these assays have throughput and resource limitations that can hamper experimental progress. The new coverslip assay outlined in this study combines the benefits of microtiter and flow cell assays and also integrates a biotic substratum for optimal S. aureus attachment. We established that the new coverslip assay is effective for monitoring biofilm formation by multiple, wild-type MRSA isolates. Mutant strains with known biofilm phenotypes also behaved as anticipated, demonstrating the new coverslip assay is robust and provides results consistent with other assays. The ability to form a biofilm showed a strong dependence on plasma coating of the coverslip, which parallels observations made with microtiter plate assays. The addition of human matrix proteins could facilitate studies on adherence proteins, transcriptional regulators, and other virulence factors that have potential roles in biofilm maturation and dispersal. Transitioning the coverslip biofilms to CLSM was also uncomplicated, providing another means to visualize biofilm phenotypes. Additionally, various surfaces such as glass, polycarbonate, or metals such as stainless steel or aluminum can potentially be substituted for the polyvinyl plastic coverslips to adapt this method to other relevant conditions, although many of these surfaces prevent efficient microscopic analysis. Altogether, the new coverslip method is a straightforward approach to quantifying and imaging $S$. aureus biofilms on biotic surfaces that should facilitate future studies in this important area of research.

\section{ACKNOWLEDGMENTS}

We thank the Inflammation Program at the University of Iowa for supplying human plasma. Jennifer N. Walker was supported by an American Heart Association predoctoral fellowship. Research in the laboratory of Alexander R. Horswill was supported by grant AI083211 from the National Institute of Allergy and Infectious Diseases.

antibiotic era. Nat. Rev. Microbiol. 7, 629-641.

Costerton, J. W. (2005). Biofilm theory can guide the treatment of devicerelated orthopaedic infections. Clin. Orthop. Relat. Res. 437, 7-11.

Cramton, S. E., Gerke, C., Schnell, N. F., Nichols, W. W., and Gotz, F. (1999). The intercellular adhesion (ica) locus is present in Staphylococcus aureus and is required for biofilm formation. Infect. Immun. 67, 5427-5433.

del Pozo, J. L., and Patel, R. (2007). The challenge of treating biofilmassociated bacterial infections. Clin. Pharmacol. Ther. 82, 204-209.

DeLeo, F. R., and Chambers, H. F. (2009). Reemergence of antibiotic-resistant Staphylococcus aureus in the genomics era. J. Clin. Invest. 119, 2464-2474.

Foster, T. J., and Hook, M. (1998). Surface protein adhesins of Staphylococcus aureus. Trends Microbiol. 6, 484-488.

Francois, P., Schrenzel, J., StoermanChopard, C., Favre, H., Herrmann, M., Foster, T. J., Lew, D. P., and Vaudaux, P. (2000). Identification of plasma proteins adsorbed on hemodialysis tubing that promote Staphylococcus aureus adhesion. J. Lab. Clin. Med. 135, 32-42.

Francois, P., Vaudaux, P., and Lew, P. D. (1998). Role of plasma and extracellular matrix proteins in the 
physiopathology of foreign body infections. Ann. Vasc. Surg. 12, $34-40$.

Goeres, D. M., Hamilton, M. A., Beck, N. A., Buckingham-Meyer, K., Hilyard, J. D., Loetterle, L. R., Lorenz, L. A., Walker, D. K., and Stewart, P. S. (2009). A method for growing a biofilm under low shear at the air-liquid interface using the drip flow biofilm reactor. Nat. Protoc. 4, 783-788.

Gotz, F. (2002). Staphylococcus and biofilms. Mol. Microbiol. 43, 1367-1378.

Guiton, P. S., Hung, C. S., Kline, K. A., Roth, R., Kau, A. L., Hayes, E., Heuser, J., Dodson, K. W., Caparon, M. G., and Hultgren, S. J. (2009). Contribution of autolysin and Sortase a during Enterococcus faecalis DNA-dependent biofilm development. Infect. Immun. 77, 3626-3638.

Hall-Stoodley, L., and Stoodley, P. (2005). Biofilm formation and dispersal and the transmission of human pathogens. Trends Microbiol. 13, 7-10.

Kiedrowski, M. R., and Horswill, A. R. (2011). New approaches for treating staphylococcal biofilm infections. Ann. N.Y. Acad. Sci. 1241, 104-121.

Kiedrowski, M. R., Kavanaugh, J. S., Malone, C. L., Mootz, J. M., Voyich, J. M., Smeltzer, M. S., Bayles, K. W., and Horswill, A. R. (2011). Nuclease modulates biofilm formation in community-associated methicillin-resistant Staphylococcus aureus. PLoS One 6:e26714. doi: 10.1371/journal.pone.0026714

Lauderdale, K. J., Boles, B. R., Cheung, A. L., and Horswill, A. R. (2009). Interconnections between Sigma B, agr, and proteolytic activity in Staphylococcus aureus biofilm maturation. Infect. Immun. 77, 1623-1635.

Lauderdale, K. J., Malone, C. L., Boles, B. R., Morcuende, J., and
Horswill, A. R. (2010). Biofilm dispersal of community-associated methicillin-resistant Staphylococcus aureus on orthopedic implant material. J. Orthop. Res. 28, 55-61.

Lowy, F. D. (1998). Staphylococcus aureus infections. N. Engl. J. Med. $339,520-532$.

Mootz, J. M., and Horswill, A. R (2012). Unpublished.

Parsek, M. R., and Singh, P. K. (2003). Bacterial biofilms: an emerging link to disease pathogenesis. Annu. Rev. Microbiol. 57, 677-701.

Shanks, R. M., Donegan, N. P., Graber, M. L., Buckingham, S. E., Zegans, M. E., Cheung, A. L., and O'Toole, G. A. (2005). Heparin stimulates Staphylococcus aureus biofilm formation. Infect. Immun. 73, 4596-4606.

Zimmerli, W., Trampuz, A., and Ochsner, P. E. (2004). Prostheticjoint infections. N. Engl. J. Med. 351, 1645-1654.
Conflict of Interest Statement: The authors declare that the research was conducted in the absence of any commercial or financial relationships that could be construed as a potential conflict of interest.

Received: 06 January 2012; accepted: 12 March 2012; published online: 28 March 2012.

Citation: Walker $J N$ and Horswill AR (2012) A coverslip-based technique for evaluating Staphylococcus aureus biofilm formation on human plasma. Front. Cell. Inf. Microbio. 2:39. doi: 10.3389/fcimb. 2012.00039

Copyright (c) 2012 Walker and Horswill. This is an open-access article distributed under the terms of the Creative Commons Attribution Non Commercial License, which permits non-commercial use, distribution, and reproduction in other forums, provided the original authors and source are credited. 